PROCEEDINGS OF THE

AMERICAN MATHEMATICAL SOCIETY

Volume 132, Number 11, Pages 3423-3431

S 0002-9939(04)07458-1

Article electronically published on May 20, 2004

\title{
MAYBERRY-MURASUGI'S FORMULA FOR LINKS IN HOMOLOGY 3-SPHERES
}

\author{
JOAN PORTI
}

(Communicated by Ronald A. Fintushel)

\begin{abstract}
We prove the Mayberry-Murasugi formula for links in homology 3 -spheres, which was proved before only for links in the 3-sphere. Our proof uses Franz-Reidemeister torsions.
\end{abstract}

\section{INTRODUCTION}

Fox's formula computes the order of the first homology group of a finite cyclic covering of a knot in $S^{3}$ from its Alexander polynomial [Fo. This formula has been generalized by Mayberry and Murasugi for finite abelian coverings of links in $S^{3}$ [MM]. Here we give a new proof of this formula using Franz-Reidemeister torsions, which applies to links not only in $S^{3}$ but in homology 3-spheres. Results in this direction have been obtained in $\mathrm{Sa}$ and $[\mathrm{HS}$.

Let $M^{3}$ be a closed three-dimensional homology sphere and $L \subset M^{3}$ a smooth link with $\mu$ components $l_{1}, \ldots, l_{\mu}$. Its exterior is denoted by $E(L)=M^{3}-N(L)$. A finite abelian covering $\hat{M}_{\pi}^{3} \rightarrow M^{3}$ branched along $L$ is given by the kernel of an epimorphism

$$
\pi: \pi_{1} E(L) \rightarrow G,
$$

where $G$ is a finite abelian group. The set of representations $\xi: G \rightarrow \mathbf{C}^{*}$ from $G$ to nonzero complex numbers is denoted by $\hat{G}$, and it is a group isomorphic to $G$, called the Pontrjagin dual.

We choose meridians $m_{1}, \ldots, m_{\mu} \in H_{1}(E(L), \mathbb{Z})$. For $\xi \in \hat{G}$, let $L_{\xi} \subseteq L$ be the sublink consisting of those components $l_{i}$ such that $\xi\left(m_{i}\right) \neq 1$. Let $\Delta_{L_{\xi}}\left(t_{i_{1}}, \ldots, t_{i_{k}}\right)$ denote the Alexander polynomial of $L_{\xi}$ (where $L_{\xi}=l_{i_{1}} \cup \cdots \cup l_{i_{k}}$ ).

For the trivial representation $\mathbf{1}: G \rightarrow \mathbf{C}^{*}, L_{\mathbf{1}}=\emptyset$, and we set $\Delta_{L_{\mathbf{1}}}=1$. Let $\hat{G}^{(1)}$ be the subset of representations $\xi \in \hat{G}$ such that $L_{\xi}$ consists of a single component: $L_{\xi}=l_{i(\xi)}$.

Finally, $\left|H_{1}\left(\hat{M}_{\pi}^{3}, \mathbf{Z}\right)\right|$ denotes the cardinality of $H_{1}\left(\hat{M}_{\pi}^{3}, \mathbf{Z}\right)$ when it is finite, or zero when it is infinite. For a complex number $z \in \mathbb{C},|z|$ denotes its modulus. The extension of the Mayberry-Murasugi formula to homology spheres is the following.

Received by the editors June 14, 2003 and, in revised form, July 1, 2003.

2000 Mathematics Subject Classification. Primary 57M12, 57Q10; Secondary 57M25, 20 K01.

Key words and phrases. Branched coverings, Franz-Reidemeister torsion.

This work was partially supported by DGICYT through grant BFM2000-0007.

(C)2004 American Mathematical Society 
Theorem 1.1. In the situation described above we have

$$
\left|H_{1}\left(\hat{M}_{\pi}^{3}, \mathbf{Z}\right)\right|=\prod_{\xi \in \hat{G}}\left|\Delta_{L_{\xi}}\left(\xi\left(m_{i_{1}}\right), \ldots, \xi\left(m_{i_{k}}\right)\right)\right| \frac{|G|}{\prod_{\xi \in \hat{G}^{(1)}}\left|1-\xi\left(m_{i(\xi)}\right)\right|} .
$$

The relationship between the Alexander polynomial and Franz-Reidemeister torsion was discovered by Milnor in M2 and further developed by Turaev T], who provided new proofs for classical results. In particular [T] reproved Fox's formula for knots in homology spheres, but not Mayberry-Murasugi's, which was said to require additional considerations going beyond the scope of the paper [T].

\section{FranZ-REIDEMEISTER TORSION}

We review the basic notions and results about Franz-Reidemeister torsion needed in this paper. See [M3] and $[\mathrm{T}$ for details.

2.1. Torsion of a chain complex. Let $F$ be a field and $C_{n} \stackrel{\partial}{\rightarrow} C_{n-1} \stackrel{\partial}{\rightarrow} \cdots \stackrel{\partial}{\rightarrow} C_{0}$ a chain complex of finite-dimensional $F$-vector spaces. Choose $c_{i}$ a basis for $C_{i}$ and $h_{i}$ a basis for the $i$-th homology group. We shall define the torsion of $C_{i}$ with respect to these bases.

Choose $b_{i}$ a basis for the $i$-dimensional boundary space (the image of $\partial: C_{i+1} \rightarrow$ $C_{i}$ ) and a lift $\tilde{b}_{i}$, which is a subset of $C_{i+1}$ such that $\partial \tilde{b}_{i}=b_{i}$. It is easy to check that the union $b_{i} \cup h_{i} \cup \tilde{b}_{i-1}$ is a basis for $C_{i}$. Let $\left[b_{i} h_{i} \tilde{b}_{i-1} / c_{i}\right] \in F^{*}$ denote the determinant of the transition matrix between both bases (its entries are the coordinates of vectors in $b_{i} \cup h_{i} \cup \tilde{b}_{i_{1}}$ with respect to $c_{i}$ ). We define

$$
\tau\left(C_{*} ; c_{i}, h_{i}\right)=\prod_{i=0}^{n}\left[b_{i} h_{i} \tilde{b}_{i-1} / c_{i}\right]^{(-1)^{i+1}} \in F^{*} /\{ \pm 1\} .
$$

It can be checked that this torsion is independent of the choice of the $b_{i}$, and it is well defined up to sign. In addition, if we change the bases $c_{i}$ and $h_{i}$, we get

$$
\tau\left(C_{*} ; c_{i}^{\prime}, h_{i}^{\prime}\right)=\tau\left(C_{*} ; c_{i}, h_{i}\right) \prod_{i=0}^{n}\left(\frac{\left[h_{i}^{\prime} / h_{i}\right]}{\left[c_{i}^{\prime} / c_{i}\right]}\right)^{(-1)^{i+1}} .
$$

Notice that we follow the convention of [M2] and [T] for the sign $(-1)^{i+1}$, opposite to the one of [M3].

2.2. Torsion of a cell complex. Let $K$ be a finite $C W$-complex and $\varphi: \pi_{1} K \rightarrow$ $F^{*}$ a representation. We define the complex with coefficients twisted by $\varphi$ to be

$$
C_{*}(K ; \varphi)=C_{*}(\tilde{K} ; \mathbf{Z}) \otimes_{\varphi} F,
$$

where $C_{*}(\tilde{K} ; \mathbf{Z})$ is the complex with integer coefficients on the universal covering. When $\varphi=\mathbf{1}$ is the trivial representation, $C_{*}(K ; \mathbf{1})=C_{*}(K ; F)$ is the usual untwisted complex.

We now choose a canonical basis for $C_{i}(K ; \varphi)$, which will play the role of $c_{i}$ in the definition of torsion. Let $\left\{e_{1}^{i}, \ldots, e_{j(i)}^{i}\right\}$ be the $i$-dimensional cells of $K$. We lift them to the universal covering, and we take $c_{i}=\left\{\tilde{e}_{1}^{i} \otimes 1, \ldots, \tilde{e}_{j(i)}^{i} \otimes 1\right\}$. The basis $c_{i}$ is called a canonical basis. Choosing again a basis $h_{i}$ for the homology, we define

$$
\tau\left(K ; \varphi, h_{i}\right)=\tau\left(C_{*}(K ; \varphi) ; c_{i}, h_{i}\right) \in F^{*} / \pm \varphi\left(\pi_{1} K\right) .
$$

This definition only depends on the combinatorial class of $K, \varphi$ and the $h_{i}$. 
Remark 2.1. We add the indeterminacy $\varphi\left(\pi_{1} K\right)$ due to the choice of the lift of cells $\tilde{e}_{j}^{i}$. Turaev avoids this indeterminacy by using Euler structures.

Example 2.2. Let $N^{n}$ be an $n$-dimensional rational homology sphere, so that $H_{i}\left(N^{n} ; \mathbf{Z}\right)$ is finite for $1 \leq i \leq n-1$. Let $h_{n} \in H_{n}\left(N^{n}, \mathbf{Z}\right)$ denote the fundamental class, and let $h_{0}$ be a generator for $H_{0}\left(N^{n}, \mathbf{Z}\right)$. For the trivial representation $\mathbf{1}: \pi_{1} N^{n} \rightarrow \mathbf{C}$ we have [T]

$$
\tau\left(N^{n} ; \mathbf{1}, h_{0}, h_{n}\right)= \pm \prod_{i=1}^{n-2}\left|H_{i}\left(N^{n} ; \mathbf{Z}\right)\right|^{(-1)^{i+1}} .
$$

Example 2.3. Let $L$ be a link in a 3-dimensional integer homology sphere $M^{3}$ with $\mu$ components. Consider its exterior $E(L)=M^{3}-N(L)$. We view the group ring $\mathbf{C}\left[\mathbf{Z}^{\mu}\right]$ as the Laurent polynomial ring with $\mu$ variables $\mathbf{C}\left[t_{1}^{ \pm 1}, \ldots, t_{\mu}^{ \pm 1}\right]$, and we let $F=\mathbf{C}\left(t_{1}, \ldots, t_{\mu}\right)$ denote its fraction field. Consider the representation induced by abelianization, $\rho: \pi_{1} E(L) \rightarrow H_{1}(E(L) ; \mathbf{Z}) \cong \mathbf{Z}^{\mu} \hookrightarrow F$. Suppose that the Alexander polynomial of the link $\Delta_{L}$ is nonzero (which is always the case for a knot). Then $C_{*}(E(L) ; \rho)$ is acyclic, and

$$
\tau(E(L) ; \rho)=\left\{\begin{array}{cc}
\Delta_{L}\left(t_{1}, \ldots, t_{\mu}\right) & \text { when } \mu>1 \\
\frac{\Delta\left(t_{1}\right)}{t_{1}-1} & \text { when } \mu=1
\end{array}\right.
$$

This was proved by Milnor when $\mu=1$ and Turaev when $\mu>1$, M2, T. Notice that those identities hold true up to multiplication by a factor $\pm t_{1}^{\alpha_{1}} \cdots t_{\mu}^{\alpha_{\mu}}$. The complex $C_{*}(E(L) ; \rho)$ has nontrivial first homology precisely when $\Delta_{L}=0$. So the formula holds true if we define $\tau(E(L) ; \rho)=0$ when the complex is not acyclic.

2.3. The order of a module over a Noetherian UFD. Both examples above can be deduced from a theorem of Turaev, since both rings $\mathbf{Z}$ and $\mathbf{C}\left[t_{1}^{ \pm 1}, \ldots, t_{\mu}^{ \pm 1}\right]$ are Noetherian unique factorization domains (UFD).

Let $R$ be a Noetherian UFD and $D$ a finitely generated $R$-module. The module $D$ has a presentation matrix, with $m$ rows and $n$ columns, where $n$ is the rank. We can always assume $m \geq n$, by adjoining rows of zeros if necessary. The elementary ideal is the ideal generated by the minors of the presentation matrix of size $n$, and the order of $D$ is the greatest common divisor of this elementary ideal. We denote it by $|D|$.

For instance, when $R=\mathbf{Z},|D|$ is the cardinality of $D$ when finite or 0 when infinite. For a link in a homology sphere, $\Delta_{L}=\left|H_{1}(\widetilde{E(L)}, \mathbf{Z})\right|$, where $\widetilde{E(L)}$ is the maximal abelian covering of the exterior of the link.

Theorem 2.4 ([T]). Let $C_{*}$ be a complex of free $R$-modules, and let $F$ be the fraction field of $R$. Then $C_{*} \otimes_{R} F$ is acyclic iff $\left|H_{i}(C)\right| \neq 0, \forall i=0, \ldots, n$. In this case

$$
\tau\left(C_{*} ; c_{i}\right)=\prod_{i=0}^{m}\left|H_{i}\left(C_{*}\right)\right|^{(-1)^{i+1}}
$$

Notice that for a link exterior $\left|H_{0}(\widetilde{E(L)}, \mathbf{Z})\right|=(t-1)$ when $\mu=1$, and $\left|H_{0}(\widetilde{E(L)}, \mathbf{Z})\right|=1$ when $\mu>1$. 


\section{Decomposing the $G$-Complex $C_{*}(\hat{K}, \mathbf{C})$}

Some of the material of sections 3 and 4 is contained in $\mathrm{Sa}$. In particular, most of the results are contained there, but we give them again for completeness and for fixing notation.

We use the notation of the introduction. Choose $K$ a CW-complex such that $|K|=M^{3}$ and $L$ is a subset of the 1-skeleton. Let $\hat{K}$ be the induced CWdecomposition of $\hat{M}_{\pi}^{3}$. By Example 2.2, $\left|H_{1}\left(\hat{M}_{\pi} ; \mathbf{Z}\right)\right|=\tau\left(\hat{K} ; \mathbf{1}, \hat{h}_{0}, \hat{h}_{3}\right)$, where $\hat{h}_{0}$ and $\hat{h}_{3}$ are $\mathbf{Z}$-bases for $H_{0}\left(\hat{M}_{\pi}^{3} ; \mathbf{Z}\right)$ and $H_{3}\left(\hat{M}_{\pi}^{3} ; \mathbf{Z}\right)$ respectively. Hence we want to study the chain complex $C_{*}(\hat{K} ; \mathbf{1})=C_{*}(\hat{K} ; \mathbf{C})$.

When the group ring $\mathbf{C}[G]$ is viewed as a $G$-module, it decomposes as a direct sum according to its representations

$$
\mathbf{C}[G]=\bigoplus_{\xi \in \hat{G}} \mathbf{C}\left[\mathbf{f}_{\xi}\right]
$$

where $\mathbf{f}_{\xi}=\frac{1}{|G|} \sum_{g \in G} \xi\left(g^{-1}\right) g \in \mathbf{C}[G]$ (cf. [Se] $)$. The element $\mathbf{f}_{\xi} \neq 0$ satisfies $\mathbf{f}_{\xi}^{2}=\mathbf{f}_{\xi}$ and $g \mathbf{f}_{\xi}=\xi(g) \mathbf{f}_{\xi}$. Thus $\mathbf{C}\left[\mathbf{f}_{\xi}\right]$ is a one-dimensional $\mathbf{C}$-vector space, isomorphic to the $G$-module associated to $\xi: G \rightarrow \mathbf{C}^{*}$.

The group $G$ acts naturally on the complex $C_{*}(\hat{K} ; \mathbf{C})$. Thus we have a decomposition of chain complexes

$$
C_{*}(\hat{K} ; \mathbf{C})=\bigoplus_{\xi \in \hat{G}} \mathbf{f}_{\xi} C_{*}(\hat{K} ; \mathbf{C}) .
$$

Next we identify each subcomplex $\mathbf{f}_{\xi} C_{*}(\hat{K} ; \mathbf{C})$, starting with the trivial representation 1 .

Lemma 3.1. There is a natural isomorphism $\mathbf{f}_{\mathbf{1}} C_{*}(\hat{K} ; \mathbf{C}) \cong C_{*}(K ; \mathbf{1})=C_{*}(K ; \mathbf{C})$.

Proof. We have a natural projection $C_{*}(\hat{K} ; \mathbf{C}) \rightarrow C_{*}(K ; \mathbf{C})$ that restricts to $\mathbf{f}_{\mathbf{1}} C_{*}(\hat{K} ; \mathbf{C}) \rightarrow C_{*}(K ; \mathbf{C})$. To construct its inverse, we map a chain $c \in C_{*}(K ; \mathbf{C})$ to $\mathbf{f}_{\mathbf{1}} \hat{c}$, where $\hat{c}$ is any lift of $c$. Since multiplication by $\mathbf{f}_{\mathbf{1}}=\frac{1}{|G|} \sum g$ is an average, this construction does not depend on the lift, and it is easily checked to be the inverse.

Since the isomorphism of Lemma 3.1 is natural, it induces an isomorphism in homology. Combining it with the decomposition (3.1), we get

Corollary 3.2. The covering $\hat{M}_{\pi}^{3}$ is a rational homology sphere iff $\mathbf{f}_{\xi} C_{*}(\hat{K} ; \mathbf{C})$ has trivial first homology group for every $\xi \in \hat{G}, \xi \neq \mathbf{1}$.

We view $K-L_{\xi}$ as a cell decomposition of the pair $\left(E\left(L_{\xi}\right), \partial E\left(L_{\xi}\right)\right)$, so that $\widetilde{K-L_{\xi}}$ is a cell decomposition of $\left(\widetilde{E\left(L_{\xi}\right)}, \partial \widetilde{E\left(L_{\xi}\right)}\right)$. The representation $\xi: G \rightarrow \mathbf{C}^{*}$ induces a representation $\pi_{1} E\left(L_{\xi}\right) \rightarrow \mathbf{C}^{*}$, also denoted by $\xi$, so that we can consider the complex:

$$
C_{*}\left(K-L_{\xi} ; \xi\right)=C_{*}\left(\widetilde{K-L_{\xi}} ; \mathbf{Z}\right) \otimes_{\xi} \mathbf{C} .
$$

Lemma 3.3. The complex $C_{*}\left(K-L_{\xi} ; \xi\right)$ is naturally isomorphic to $\mathbf{f}_{\xi} C_{*}(\hat{K} ; \mathbf{C})$.

Proof. The projection $\widetilde{K-L_{\xi}} \rightarrow \hat{K}$ induces a natural map $C_{*}\left(K-L_{\xi} ; \xi\right) \rightarrow$ $\mathbf{f}_{\xi} C_{*}(\hat{K} ; \mathbf{C})$. It is straightforward to check that it is well defined. Before constructing the inverse, notice that if $\hat{e}_{j}^{i}$ is a cell of $\hat{K}$ that projects to $L_{\xi}$, then there 
exists a $g \in G$ (the image of its meridian) such that $g \hat{e}_{j}^{i}=\hat{e}_{j}^{i}$ and $\xi(g) \neq 1$. Thus $\mathbf{f}_{\xi} \hat{e}_{j}^{i}=\mathbf{f}_{\xi} g \hat{e}_{j}^{i}=\xi(g) \mathbf{f}_{\xi} \hat{e}_{j}^{i}=0$. This shows that we can construct a map just by taking lifts of chains, which is easily checked to be the inverse.

\section{The NON-ACYCLIC CASE}

Lemma 4.1. The homology of $\mathbf{f}_{\xi} C_{*}(\hat{K} ; \mathbf{C})$ is isomorphic to $H_{*}\left(E\left(L_{\xi}\right) ; \xi\right)$.

Proof. By Lemma 3.3 the homology of the complex $\mathbf{f}_{\xi} C_{*}(\hat{K} ; \mathbf{C})$ is isomorphic to $H_{*}\left(E\left(L_{\xi}\right), \partial E\left(L_{\xi}\right) ; \xi\right)$. Using the exact sequence of the pair, it suffices to prove that $H_{*}\left(\partial E\left(L_{\xi}\right) ; \xi\right)=0$. Notice that $\partial E\left(L_{\xi}\right)$ is a union of 2-dimensional tori, such that the restriction of $\xi$ to each component is nontrivial. This implies that $H^{0}\left(\partial E\left(L_{\xi}\right) ; \xi\right)=0$, because for each component, the 0-cohomology group gives the subspace invariant under the representation. A standard argument using duality and the Euler characteristic proves the claim.

Proposition 4.2. For $\xi \in \hat{G}, \xi \neq 1$,

$$
H_{1}\left(E\left(L_{\xi}\right) ; \xi\right)=0 \quad \text { iff } \quad \Delta_{L_{\xi}}\left(\xi\left(m_{i_{1}}\right), \ldots, \xi\left(m_{i_{k}}\right)\right) \neq 0 .
$$

This proposition is already proved in $\left[\mathrm{Sa}\right.$ and $[\mathrm{HS}]$ using the pair $\left(E\left(L_{\xi}\right), *\right)$, where $* \in E\left(L_{\xi}\right)$ is a point. Here we give a proof using the formalism of torsions.

Proof. Consider the representation $\rho$ of $\pi_{1} E\left(L_{\xi}\right)$ induced by abelianization as in Example 2.3 Let $W$ be a 2-dimensional $C W$-complex with the homotopy type of $E\left(L_{\xi}\right)$, so that $C_{*}(W ; \xi)$ and $C_{*}(W ; \rho)$ give the homology of $E\left(L_{\xi}\right)$ with coefficients $\xi$ and $\rho$ respectively. To distinguish tensor products, we write $\otimes_{\xi}$ and $\otimes_{\rho}$.

Assuming that $W$ has a single 0 -cell, let $e_{1}^{2}, \ldots, e_{k}^{2}$ be the 2 -cells of $W, e_{1}^{1}, \ldots, e_{k+1}^{1}$ its 1-cells and $e^{0}$ its 0 -cell. By lifting these cells, we construct canonical bases $c_{i}(\xi)$ and $c_{i}(\rho)$ for $C_{i}(W ; \xi)$ and $C_{i}(W ; \rho)$ respectively, as in Subsection 2.1

Since $H_{0}\left(E\left(L_{\xi}\right) ; \mathbf{C}\right)=0$, up to reindexing we may assume that $\partial\left(\tilde{e}_{k+1}^{1} \otimes_{\xi} 1\right) \neq 0$. Hence $\tilde{e}_{k+1}^{1} \otimes_{\xi} 1$ is not contained in the image of $\partial: C_{2}(W ; \xi) \rightarrow C_{1}(W ; \xi)$, and

$$
\begin{aligned}
H_{1}\left(E\left(L_{\xi}\right) ; \xi\right)=0 & \Leftrightarrow \operatorname{rank}_{\mathbf{C}}\left(\partial: C_{2}(W ; \xi) \rightarrow C_{1}(W ; \xi)\right)=k \\
& \Leftrightarrow \partial c_{2}(\xi) \cup\left\{\tilde{e}_{k+1}^{1} \otimes_{\xi} 1\right\} \text { is a basis for } C_{1}(W ; \xi) \\
& \Leftrightarrow \frac{\left[\partial c_{2}(\xi) \cup\left\{\tilde{e}_{k+1}^{1} \otimes_{\xi} 1\right\} / c_{1}(\xi)\right]}{\left[\partial\left(\tilde{e}_{k+1}^{1} \otimes_{\xi} 1\right) / \tilde{e}^{0} \otimes_{\xi} 1\right]} \neq 0 .
\end{aligned}
$$

Consider the evaluation map $\epsilon_{\xi}: \mathbf{C}\left[t_{i_{1}}^{ \pm 1}, \ldots, t_{i_{k}}^{ \pm 1}\right] \rightarrow \mathbf{C}$, i.e., $\epsilon_{\xi}\left(p\left(t_{i_{1}}, \ldots, t_{i_{k}}\right)\right)=$ $p\left(\xi\left(m_{i_{1}}\right), \ldots, \xi\left(m_{i_{k}}\right)\right)$. This map obviously extends to quotients of polynomials $p / q$ such that $\epsilon_{\xi}(q) \neq 0$. Since $\left[\partial\left(\tilde{e}_{k+1}^{1} \otimes_{\xi} 1\right) / \tilde{e}^{0} \otimes_{\xi} 1\right] \neq 0$, we deduce that

$$
\frac{\left[\partial c_{2}(\xi) \cup\left\{\tilde{e}_{k+1}^{1} \otimes_{\xi} 1\right\} / c_{1}(\xi)\right]}{\left[\partial\left(\tilde{e}_{k+1}^{1} \otimes_{\xi} 1\right) / \tilde{e}^{0} \otimes_{\xi} 1\right]}=\epsilon_{\xi}\left(\frac{\left[\partial c_{2}(\rho) \cup\left\{\tilde{e}_{k+1}^{1} \otimes_{\rho} 1\right\} / c_{1}(\rho)\right]}{\left[\partial\left(\tilde{e}_{k+1}^{1} \otimes_{\rho} 1\right) / \tilde{e}^{0} \otimes_{\rho} 1\right]}\right) .
$$

Notice that by construction this last term is $\epsilon_{\xi}\left(\tau\left(E\left(L_{\xi}\right) ; \rho\right)\right)$. This equality also holds true when using the convention $\tau\left(E\left(L_{\xi}\right) ; \rho\right)=0$ if $H_{1}\left(E\left(L_{\xi}\right) ; \rho\right) \neq 0$. Hence, by Example 2.3 $H_{1}\left(E\left(L_{\xi}\right) ; \xi\right)=0$ iff $\epsilon_{\xi}\left(\Delta_{L_{\xi}}\right) \neq 0$, as claimed.

The following corollary is obtained in [Sa], where a formula for the first Betti number is given. Here it follows from Corollary[3.2, Lemma 4.1] and Proposition 4.2 and proves the non-acyclic case of Theorem 1.1 . 
Corollary 4.3. The covering $\hat{M}_{\pi}^{3}$ is a rational homology sphere if and only if $\Delta_{L_{\xi}}\left(\xi\left(m_{i_{1}}\right), \ldots, \xi\left(m_{i_{k}}\right)\right) \neq 0$ for all $\xi \in \hat{G}$.

\section{Proof of the main theorem}

The strategy of the proof is as follows. The order of $H_{1}\left(\hat{M}_{\pi}^{3} ; \mathbf{Z}\right)$ is the torsion of the complex $C_{*}(\hat{K} ; \mathbf{C})$. We use the decomposition (3.1) to write this torsion as a product of torsions of the complexes $\mathbf{f}_{\xi} C_{*}(\hat{K} ; \mathbf{C})$ (Formula (5.1) below). To get this formula, we change the canonical basis for $C_{*}(\hat{K} ; \mathbf{C})$ to a union of canonical bases for $\mathbf{f}_{\xi} C_{*}(\hat{K} ; \mathbf{C})$ (this is done in Subsection 5.1). In Subsection 5.2 we compute the torsion of each complex in terms of Alexander polynomials. All computations in Subsection 5.2 have an indeterminacy of roots of unity, since the torsions we compute are defined up to some root of unity and the Alexander polynomial is defined up to some factor $t_{1}^{\alpha_{1}} \cdots t_{\mu}^{\alpha_{\mu}}$. This indeterminacy is eliminated by taking absolute values in Theorem 1.1.

5.1. Changing the canonical basis. Let $\left\{e_{j}^{i} \mid i=0,1,2,3\right.$ and $\left.j=1, \ldots, j(i)\right\}$ denote the set of cells of $K$. Choose lifts $\hat{e}_{j}^{i}$ to $\hat{K}$, so that, for $i=0,1,2,3$,

$$
c_{i}=\left\{g \hat{e}_{j}^{i} \mid j=1, \ldots, j(i) \text { and } g \in G / \operatorname{Stab}\left(\hat{e}_{j}^{i}\right)\right\}
$$

is the set of $i$-cells of $\hat{K}$, and hence a canonical basis for $C_{i}(\hat{K} ; \mathbf{C})$. Define

$$
c_{i}^{\prime}(\xi)=\left\{\mathbf{f}_{\xi} \hat{e}_{j}^{i} \mid j=1, \ldots, j(i) \text { and }\left.\xi\right|_{S t a b\left(\hat{e}_{j}^{i}\right)} \text { is trivial }\right\} .
$$

Lemma 5.1. The isomorphism of Lemma 3.1 maps $c_{i}^{\prime}(\mathbf{1})$ to a canonical basis for $C_{i}(K ; \mathbf{C})=C_{i}(K ; \mathbf{1})$. The one of Lemma 3.3 maps $c_{i}^{\prime}(\xi)$ to a canonical basis for $C_{i}\left(K-L_{\xi} ; \xi\right)$.

Proof. A direct computation shows that $\mathbf{f}_{\xi} \hat{e}_{j}^{i}$ is mapped to $\tilde{e}_{j}^{i} \otimes_{\xi} 1$ when $\xi \neq \mathbf{1}$, and to $e_{j}^{i}$ when $\xi=\mathbf{1}$. Counting elements, we realize that this is a canonical basis.

In particular, $c_{i}^{\prime}(\xi)$ is a basis for $\mathbf{f}_{\xi} C_{i}(\hat{K} ; \mathbf{C})$ and $\bigcup_{\xi \in \hat{G}} c_{i}^{\prime}(\xi)$ is a basis for $C_{i}(\hat{K} ; \mathbf{C})$.

$$
\text { Lemma 5.2. } \prod_{i=0}^{3}\left[\bigcup_{\xi \in \hat{G}} c_{i}^{\prime}(\xi) / c_{i}\right]^{(-1)^{i}}=1 \text {. }
$$

Proof. For each subgroup $H<G$, the set of lifts $\left\{\hat{e}_{j}^{i}\right\}$ of cells that have precisely $H$ as stabilizer has zero Euler characteristic. This implies that there are cancellations in the alternated product.

When computing the torsion of the complexes $C_{i}(K ; \mathbf{C})$ and $C_{i}\left(K-L_{\xi} ; \xi\right)$, we will assume that we are using the canonical basis of Lemma 5.1

It follows from the decomposition (3.1) and from Lemmas 3.1, 3.3 and 5.2 that

$$
\tau\left(\hat{K} ; \mathbf{1}, \hat{h}_{0}, \hat{h}_{3}\right)=\tau\left(M^{3} ; \mathbf{1}, \hat{h}_{0}, \hat{h}_{3}\right) \prod_{\substack{\xi \in \hat{G} \\ \xi \neq 1}} \tau\left(E\left(L_{\xi}\right), \partial E\left(L_{\xi}\right) ; \xi\right) .
$$


5.2. The torsion $\tau\left(E\left(L_{\xi}\right), \partial E\left(L_{\xi}\right) ; \xi\right)$ as evaluation of the Alexander polynomial.

Lemma 5.3. $\tau\left(M^{3} ; \mathbf{1}, \hat{h}_{3}, \hat{h}_{0}\right)=|G|$.

Proof. Since the isomorphism of Lemma 3.1 is induced by the projection $\hat{M}_{\pi}^{3} \rightarrow M^{3}$, it follows that $\hat{h}_{0}$ is mapped to $h_{0}$, a generator for $H_{0}\left(M^{3} ; \mathbb{Z}\right)$, and the fundamental class $\hat{h}_{3}$ of $\hat{M}_{\pi}^{3}$ is mapped to $|G|$ times the fundamental class $h_{3}$ of $M^{3}$. Thus, by (2.1),

$$
\tau\left(M^{3} ; \mathbf{1}, \hat{h}_{3}, \hat{h}_{0}\right)=|G| \tau\left(M^{3} ; \mathbf{1}, h_{3}, h_{0}\right),
$$

and $\tau\left(M^{3} ; \mathbf{1}, h_{3}, h_{0}\right)=1$ because $M^{3}$ is a homology sphere (Example 2.2).

Lemma 5.4. Let $\xi \in \hat{G}$ with $\xi \neq 1$. Then

$$
\tau\left(E\left(L_{\xi}\right), \partial E\left(L_{\xi}\right) ; \xi\right)= \begin{cases}\tilde{\Delta}_{L}(\xi) & \text { if } \xi \notin \hat{G}^{(1)} \\ \frac{\tilde{\Delta}_{L}(\xi)}{\xi\left(m_{i(\xi)}\right)-1} & \text { if } \xi \in \hat{G}^{(1)}\end{cases}
$$

Proof. We consider the exact sequence of the pair

$$
0 \rightarrow C_{*}\left(\partial E\left(L_{\xi}\right) ; \xi\right) \rightarrow C_{*}\left(E\left(L_{\xi}\right) ; \xi\right) \rightarrow C_{*}\left(E\left(L_{\xi}\right), \partial E\left(L_{\xi}\right) ; \xi\right) \rightarrow 0 .
$$

We showed in the proof of Lemma 4.1 that $C_{*}\left(\partial E\left(L_{\xi}\right) ; \xi\right)$ is acyclic. By Sections 3 and 4 we may assume that all complexes in the sequence are acyclic. Applying [M3 Thm. 3.1] (see also [M1, Lemma 4]), we get

$$
\tau\left(E\left(L_{\xi}\right) ; \xi\right)=\tau\left(\partial E\left(L_{\xi}\right), \xi\right) \tau\left(E\left(L_{\xi}\right), \partial E\left(L_{\xi}\right) ; \xi\right) .
$$

The torsion $\tau\left(\partial E\left(L_{\xi}\right), \xi\right)$ is trivial because $\partial E\left(L_{\xi}\right)$ is even dimensional [Fr] (see also [M2]; otherwise a direct computation on the torus shows it). Thus

$$
\tau\left(E\left(L_{\xi}\right), \partial E\left(L_{\xi}\right) ; \xi\right)=\tau\left(E\left(L_{\xi}\right) ; \xi\right) .
$$

Consider the representation $\rho: \pi_{1} E\left(L_{\xi}\right) \rightarrow \mathbf{C}\left[t_{j_{1}}^{ \pm 1}, \ldots t_{j_{k}}^{ \pm 1}\right] \subset \mathbf{C}\left(t_{j_{1}}, \ldots t_{j_{k}}\right)$ corresponding to the abelianization, so that $H_{*}\left(E\left(L_{\xi}\right) ; \rho\right)=0$ and

$$
\tau\left(E\left(L_{\xi}\right) ; \rho\right)=\left\{\begin{array}{cl}
\Delta_{L}\left(t_{j_{1}}, \ldots, t_{j_{k}}\right) & \text { when } \xi \notin \hat{G}^{(1)}(\text { i.e., } k>1), \\
\frac{\Delta\left(t_{i(\xi)}\right)}{t_{i(\xi)}-1} & \text { when } \xi \in \hat{G}^{(1)} .
\end{array}\right.
$$

Representations $\xi$ and $\rho$ are related by the evaluation morphism:

$$
\begin{aligned}
\epsilon_{\xi}: \mathbf{C}\left[t_{j_{1}}^{ \pm 1}, \ldots t_{j_{k}}^{ \pm 1}\right] & \rightarrow \mathbf{C} \\
f\left(t_{j_{1}}, \ldots, t_{j_{k}}\right) & \mapsto p\left(\xi\left(m_{j_{1}}\right), \ldots, \xi\left(m_{j_{k}}\right)\right) .
\end{aligned}
$$

We have $\xi=\epsilon_{\xi} \circ \rho$. We claim that

$$
\epsilon_{\xi}\left(\tau\left(E\left(L_{\xi}\right) ; \rho\right)\right)=\tau\left(E\left(L_{\xi}\right) ; \xi\right)
$$

Before proving the claim, it is relevant to notice that $\epsilon_{\xi}$ is defined on the polynomial ring $\mathbf{C}\left[t_{j_{1}}^{ \pm 1}, \ldots t_{j_{k}}^{ \pm 1}\right]$ but not on the whole fraction field $\mathbf{C}\left(t_{j_{1}}, \ldots, t_{j_{k}}\right)$. This problem can be avoided by computing the torsion following the method of [T] or [M2]. Namely, we choose $\tilde{b}_{2}$ to be the canonical basis for $C_{3}\left(E\left(L_{\xi}\right) ; \xi\right)$. Acyclicity implies that $b_{2}=\partial \tilde{b}_{2}$ is a set of linearly independent elements in $C_{2}\left(E\left(L_{\xi}\right) ; \xi\right)$. We complete $b_{2}$ to a basis for $C_{2}\left(E\left(L_{\xi}\right) ; \xi\right)$ by choosing elements of the canonical basis, whose union we denote by $\tilde{b}_{1}$. Again acyclicity implies that $b_{1}=\partial \tilde{b}_{1}$ is a set of linearly independent elements, and so on. Each time we choose elements of the canonical basis for $\xi$, we do the corresponding choice for $\rho$. In this way, all the determinants involved in the torsion for $\rho$ belong to $\mathbf{C}\left[t_{j_{1}}^{ \pm 1}, \ldots t_{j_{k}}^{ \pm 1}\right]$ and have the property that 
$\epsilon_{\xi}$ maps them to the corresponding determinants for computing the torsion for $\xi$. Hence (5.2) follows.

\section{Generalizations}

6.1. Abelian coverings branched along graphs. Following [Sa, we can generalize Theorem 1.1 to coverings of homology three-spheres along graphs. There are some important restrictions to our graph. First, the vertices must have valency three, if we want the covering to be a manifold. In addition, the only abelian finite subgroups of $S O(3)$ are either cyclic or $\mathbf{Z} / 2 \mathbf{Z} \oplus \mathbf{Z} / 2 \mathbf{Z}$. Hence, when we have a trivalent vertex, the ramification on the adjacent edges must be 2 . Once those restrictions are established, Theorem 1.1 generalizes for $L$ to be such an embedded graph, not only a link. This is because each cyclic subgroup of $S O(3)$ fixes an edge, and even if $L$ is a graph, for every representation $\xi \in \hat{G}$, the subgraph $L_{\xi}$ is a link.

6.2. Higher-dimensional knots. We work in the PL-category. Let $M^{n+2}$ be an $(n+2)$-dimensional homology sphere and $K^{n} \subset M^{n+2}$ an $n$-knot, with $K^{n} \cong S^{n}$. We can consider the $d$-cyclic branched covering $\hat{M}_{d}^{n+2} \rightarrow M^{n+2}$ branched along $K^{n}$.

In this case, we have not only the Alexander polynomial but several Alexander invariants. The exterior $E\left(K^{n}\right)=M^{n+2}-N\left(K^{n}\right)$ has the homology of the circle, and we consider its infinite abelian covering $\widehat{E\left(K^{n}\right)}$.

The $i$-th Alexander invariant is defined to be the order of $H_{i}\left(\widetilde{E\left(K^{n}\right)} ; \mathbf{Z}\right)$ as a $\mathbf{Z}\left[t, t^{-1}\right]$-module:

$$
A_{i}(t)=\left|H_{i}\left(\widetilde{E\left(K^{n}\right)} ; \mathbf{Z}\right)\right|
$$

Theorem 6.1. The covering $M_{d}^{n+2}$ is a rational homology sphere iff $A_{i}(\zeta) \neq 0$ for every d-root of unity $\zeta$ and every $i$. When it is a rational homology sphere, we have

$$
\prod_{i=1}^{n}\left|H_{i}\left(\hat{M}_{d}^{n+2} ; \mathbf{Z}\right)\right|^{(-1)^{i+1}}=\prod_{i=1}^{n+1} \prod_{k=1}^{d} A_{i}\left(\zeta^{d}\right)^{(-1)^{i+1}},
$$

where $\zeta$ is a primitive d-root of unity.

Of course this formula is only relevant when $n$ is odd, because when $n$ is even each one of the products is 1 (the torsion of an even-dimensional manifold is trivial $\mathrm{Fr}$ ).

The proof of this theorem follows exactly the same argument as Theorem 1.1 with minor changes.

\section{ACKNOWLEDGEMENTS}

I am indebted to M. Sakuma for an encouraging conversation. I also thank the referee for useful comments.

\section{REFERENCES}

[Fo] R. H. Fox. Free differential calculus. III. Subgroups. Ann. of Math. (2) 64 (1956), 407-419. MR 20:2374

[Fr] W. Franz. Torsionsideale, Torsionsklassen und Torsion. J. Reine Angew. Math. 176 (1936), 113-124.

[HS] J. Hillman and M. Sakuma. On the homology of finite abelian coverings of links. Canad. Math. Bull. 40 (1997), no. 3, 309-315. MR 98i:57010 
[MM] J. P. Mayberry and K. Murasugi. Torsion-groups of abelian coverings of links. Trans. Amer. Math. Soc. 271 (1982), no. 1, 143-173. MR 84d:57004

[M1] J. Milnor. Two complexes which are homeomorphic but combinatorially distinct. Ann. of Math. (2) 74 (1961), 575-590. MR 24:A2961

[M2] J. Milnor, A duality theorem for Reidemeister torsion. Ann. of Math. (2) 76 (1962), 137147. MR 25:4526

[M3] J. Milnor, Whitehead torsion. Bull. Amer. Math. Soc. 72 (1966), 358-426. MR 33:4922

[Sa] M. Sakuma. Homology of abelian coverings of links and spatial graphs. Canad. J. Math. 47 (1995). MR 96d:57008

[Se] J.-P. Serre. Représentations linéaires des groupes finis. Hermann, Paris, 1967. MR 38:1190

[T] V. G. Turaev. Reidemeister torsion in knot theory. Russian Math. Surveys 41 (1986), no. 1, 119-182. MR 87i:57009

Departament de Matemàtiques, Universitat Autònoma de Barcelona, 08193 BellaTERRA, SPAIN

E-mail address: porti@mat.uab.es 